\title{
Electronic peer review? \\ (A report of the Electronic Libraries Programme ESPERE project)
}

\author{
DEE WOOD
}

ESPERE Project Manager

Salix Education

\section{Introduction}

The Electronic Submission and Peer Review project is one of approximately 60 projects funded by the Joint Information Systems Committee (JISC) of the Higher Education Funding Councils, as part of its Electronic Libraries Programme (eLib). ESPERE is particularly concerned with the possibility of using electronic methods for peer review, initially for biomedical articles. The project is jointly run by the University of Ulster and the Society for Endocrinology and has seven learned society publishers as its partners.

Peer review is an essential part of academic activity. In effect it is a quality assurance scheme which validates research by subjecting it to the scrutiny of other researchers in the same field of study. For a typical journal the peer review process starts with the receipt of an article from an author and continues with the distribution of copies of the article to at least two academics who are considered to be experts in the field of study concerned. The reports received from these referees are then considered by the editor of the journal and a decision is made as to whether the article can be accepted for publication. In fact direct acceptance is rare and very few articles are published without one, or even several, revisions. However in all cases peer review consists of the following stages:

1. Receipt of article by editor or publisher

2. Choice of referees (by the editor)

3. Mailing the papers to referees

4. Decision based on one or more referee/s' reports and the editor's judgement

5. Communicating the decision (acceptable/acceptable after revision/rejection) to the author

Keeping track of the peer review process is a complex activity, particularly if a large number of papers are published each month, since many papers will be at a different stage in the process simultaneously. Even for a small journal which is only produced quarterly it can be hard to keep track of individual papers. Authors contacting the editor are often surprised to find that he or she cannot tell them its status immediately! Most publishers use an article handling database so that they can check the status of any given paper for an individual author and maintain control over the refereeing process by producing regular reports.

Most referees either fax their reports or use electronic mail (email) and some authors are now in a position to send their articles by email. However, relatively few journal publishers in biomedical subjects are currently accepting or indeed encouraging the use of this method. There is considerable concern about the quality of graphics and also the difficulty of maintaining special characters (eg Greek letters, accented characters etc) across multiple word processors and platforms. Most journal publishers accept (some require) authors' word processor files but they are not anxious to receive these as email attachments. 


\section{ESPERE and email}

The ESPERE project was originally conceived as an email version of the current system. However, even a limited exploration into the world of email has indicated that as a medium for the exchange of documents it is not ideal. The user interface of much of the software which deals with email is poor and off-putting for all but the dedicated enthusiast. A questionnaire completed by members of eLib was hardly encouraging (Email survey 1996) ${ }^{(1)}$. Almost all the respondents had had to work hard to send documents to others, many had failed or were too concerned about failure to try and the general consensus was that it was fine between consenting parties! In other words if you find out first what someone else's system can deal with, and you have a reasonable level of expertise yourself, you can send even large files around.

This is hardly an ideal situation and though it will doubtless improve immensely over the next year, with the newer versions of browsers like Netscape and Internet Explorer becoming integrated with mail software, the number of different systems currently in use makes it difficult for the time being.

\section{The World Wide Web}

As a result of these findings the focus has moved to the user-friendly side of the Internet - the World Wide Web - as a possible interface for the transfer of articles, but also as a possible medium for the further development of the peer review system. The Web was originally used by computer scientists - often students in their early twenties - but as recent surveys have shown (Kehoe \& Pitkow 1996) (2) its use is now much more widespread across both subject specialities and age groups. It has the huge advantage of being an interactive medium. Thus information can be provided and requested. Since the nature of peer review is essentially a two way process this has considerable advantages. The ESPERE project will be looking into the use of the Web for transferring articles from authors to journals and for providing referees with access to those articles.

The spectacular growth of the World Wide Web has led to a great interest in electronic journals documents which can be accessed from the researcher's desktop - and the numbers of these journals are increasing all the time. There was some reluctance at first to submit articles to these journals, partly because the medium was new and also because they did not necessarily include a peer review process. However, the importance of this method of quality control was soon recognised (Harnad $1995)^{(3)}$ and their growth has continued. Many now operate a peer review process: Association of Research Library statistics (ARL 1991-)(4) show that the number of peer reviewed online journals and newsletters increased from 73 in 1994 to 517 in 1996 and this trend is set to continue.

A few journals, such as the Medical Journal of Australia ${ }^{(5)}$, Behaviour and Brain Sciences $^{(6)}$ and the Journal of Interactive Media in Education ${ }^{(7)}$ are experimenting with novel methods of peer review which include making articles available before publication for general comment and in some cases providing the opportunity for contributors to have a dialogue with the authors.

\section{Attitudes to electronic peer review}

Despite these interesting developments most journal articles, even those intended for online journals and particularly those that include graphics, are still supplied to the journal publisher via the postal system (now quaintly known as 'snail mail' to computer enthusiasts). Articles are posted to referees all over the world even if the reports are now sent back by email. The ESPERE project has been investigating the many aspects of the peer review system and in particular the attitudes of authors, referees and learned society publishers to electronic methods. This was done by a combination of interviews, focus group and questionnaire (ESPERE Stage One Report 1996) ${ }^{(8)}$. 
There was widespread enthusiasm for electronic peer review and both authors and referees pointed out the numerous benefits of such a system. These include a fairer and more global approach to refereeing - if it was as easy (and as fast) to ask an academic anywhere in the world to referee a paper this might raise the standard of refereeing and also give all countries equal access to journals.

The cost of providing many copies of graphical material for review was mentioned by many as a serious problem. In fact much of the graphical material is now scanned and is therefore available as electronic files and the use of digital cameras which produce photographs as electronic files is likely to increase. Many departments are maintaining photographic images on Kodak PhotoCDs and databases of images are now available through the Web.

Although few referees were keen to edit articles on screen, most were prepared to print out articles for review while they were away from their desk (referees frequently read articles while travelling). Some referees complained that the cost of this would be transferred to them. However, those who were also authors (most of them) would benefit equally by not having to supply four copies of their own article.

Publishers were also enthusiastic because of the opportunities to streamline systems. However, they realised that these developments would require some major changes and retraining of editorial staff, which would incur substantial costs even if these might be recouped later in increased efficiency.

\section{File formats}

These responses were obtained despite the fact that there was no mention of the technical details of precisely how and in what format authors might be required to submit files. The majority of authors use Word or WordPerfect as their word processor but graphics may be created in a wide variety of packages. If files are to be accessed by referees, who may be working on Macs, PCs or UNIX systems a format must be found that is suitable across these different platforms and a number of possibilities have been considered eg Word, Acrobat portable document format (PDF), and Hypertext Markup Language (HTML). All have some drawbacks, but for files which invariably contain graphics PDF may be the most suitable format for distribution to referees at present. These aspects will be further investigated in Stage 2 of the project.

\section{Stage 2}

As a result of the interest shown in electronic methods of peer review the project has now progressed to the next phase, which involves a pilot system, based on a Web interface, launched in the Autumn of 1997.

For further information please visit the ESPERE Web site http://www.ulst.ac.uk/espere

\section{References}

1. ESPERE Email survey (1996). Available from the author or at http://ulst.ac.uk/espere

2. Kehoe C.M. and Pitkow J.E. Surveying the territory: GVU's Five WWW user surveys, The World Wide Web Journal Vol 1, no 3, 1996.

3. Harnad S. Implementing peer review on the net: scientific quality control in electronic journals. In Peek R. and Newby G. (Eds) Electronic publishing confronts academia: the agenda for the year 2000. Cambridge MA: MIT Press, 1995. 
4. Directory of electronic journals, newsletters and academic discussion lists. Washington, DC: Association of Research Libraries, Office of Scientific and Academic Publishing, 1991.

5. Behaviour and Brain Sciences - http://www.princeton.edu/ harnad/bbs/index.html

6. Journal of Interactive Media in Education - http://www-jime.open.ac.uk/jime

7. Medical Journal of Australia - http://www.library.usyd.edu.mja

8. ESPERE Stage One Report (1996). Available from the author or at http://ulst.ac.uk/espere 